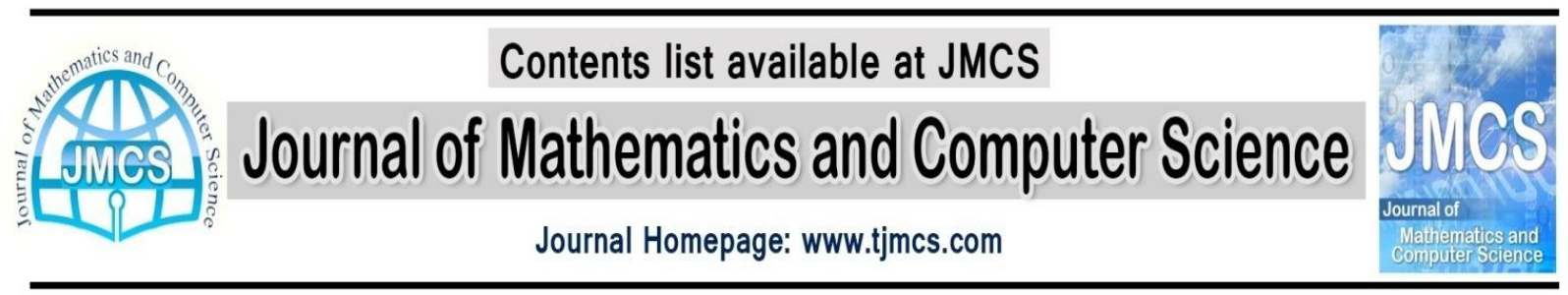

\title{
Weighted Information Function of Dynamical Systems
}

\author{
Uosef Mohamadi \\ Department of Mathematics, Faculty of Mathematics and Computer \\ University of Jiroft, Jiroft, IRAN, 78671-61167
}

Article history:
Received January 2014
Accepted March 2014
Available online March 2014

\begin{abstract}
In this paper we prove that the local information function of a dynamical systems affine. We also introduce the concept of weighted information function for continuous dynamical systems on compact metric spaces, and prove some of its properties. At the end we prove that the weighted information function is invariant under isomorphism.
\end{abstract}

KEY WORDS: Dynamical systems, generator, weighted information function, local information function, invariant.

\section{INTORODUCTION}

There is an enormous literatures on information but the 1948 paper A mathematical theory of communication by Clude Shannon [7] in the Bell System Technical Journal remains one of the most readable accounts of its properties and significance. Note that in later versions it became The mathematical theory of communication.

Then, McMillan [5] found some results relating to this function. And recently I. Guney, I. Tok and M. Yamankaradeniz [3] investigated some basic properties of the fuzzy local information function of dynamical systems.

In this study we define the weighted information function for a continuous systemT: $X \rightarrow X$ on a compact metric space $X$ and prove some important ergodic properties of this function. The idea is based on the feeling that any 'intelligent' point in the space is informed about the space, as it increases its experience by meeting different areas of the space under the dynamic of $T$. As disjoint points may have different 'intelligence', we may assign a weight factor to the local loss of information caused by the lack of experience of any point.

In this article, $M(X)$ denotes the set of all probability measures on Borel sets of $X$. The set of all probability measures on $\mathrm{X}$ preserving $T$ is denoted by $M(X, T)$. We also write $E(X, T)$ for the set of all ergodic measures of $T$.

\section{PRELIMINARIES AND METHODS}

Let $(X, \beta)$ denotes a $\sigma$-finite measure space, i.e. a set equipped with a $\sigma$-algebra $\beta$ of subsets of $X$. Further let $\mu$ denote a probability measure on $(X, \beta)$. Then $(X, \beta, \mu)$ is called a probability space. Let

Corresponding Author: Uosef Mohamadi, Email: u.mohamadi@ujiroft.ac.ir 
$T: X \rightarrow X$ be a measure-preserving invertible transformation of the probability space $(X, \beta, \mu)$. In particular $T(\beta)=\beta$ and $\mu\left(T^{-1}(A)\right)=\mu(A)$ for all $A \in \beta$. Then $(X, \beta, \mu, T)$ is called a dynamical system. In applications one also encounters dynamical systems in which $T$ is replaced by a measurepreserving flow, i.e. a one parameter family of measure-preserving transformations $\left\{T_{t}\right\}_{t \in \mathbb{R}}$ such that $T_{s} T_{t}=T_{s+t}, T_{-s}=\left(T_{s}\right)^{-1}$ and $T_{0}=I$ is the identity. The flow is usually interpreted as a description of the change with time $t$ of the observables $A$. The single automorphism $T$ can be thought of as the change with unit time and $T^{n}$ is the change after $n$-units of time. A finite partition $\alpha=\left\{A_{1}, A_{2}, \ldots, A_{n}\right\}$ of the space is the collection of a finite number of disjoint elements $A_{i}$ of $\beta$ such that $\bigcup_{i=1}^{n} A_{i}=X$. Definition 2.1 Let $\alpha=\left\{A_{1}, A_{2}, \ldots, A_{n}\right\}$ be a finite measurable partition of $X$ and $\mu \in M(X, T)$. Then if $A_{i} \in \alpha, \mathrm{i}=1,2, \ldots, \mathrm{n}$ is an observed event. The information $I(\alpha$, ? $)$ carried by $\alpha$ may be defined as; $I(\alpha$, ? $)=$ $-\log$ ? $\left(A_{i}\right)$ and the quantity $I(x, \alpha$, ? $)=-\sum_{i=1}^{n} \chi_{A_{i}}(x) \log \mu\left(A_{i}\right)$ for each $\mathrm{x} \in X$ is called local information function.

Where $\chi_{A_{i}}$ is the characteristic function of $A_{i}$ defined by $\chi_{A_{i}}(x)= \begin{cases}1 & \text { if } x \in A_{i} \\ 0 & \text { if } x \notin A_{i}\end{cases}$

Definition 2.2 Given two partitions $\alpha, \gamma$ we define their refinement

$$
\alpha \vee \gamma=\left\{A_{i} \cap B_{j}: A_{i} \in \alpha, B_{j} \in \gamma\right\}
$$

Lemma 2.3 Let $\left\{a_{n}\right\}_{n \in \mathbb{N}}$ be a sequence of real numbers such that is positive and sub additive. Then $\lim _{n \rightarrow \infty} \frac{a_{n}}{n}$ exists and is equal toinf $f_{n \in \mathbb{N}} \frac{a_{n}}{n}$.

Proof: See, the Theorem 4-9 of [8].

Theorem 2.4 Let $\alpha$ and $\gamma$ be two partitions of $X$ with $\mathrm{I}(\mathrm{x}, \alpha, \mu)<\infty$ and $\mathrm{I}(\mathrm{x}, \gamma, \mu)<\infty$, for $\mathrm{x} \in X$. Then, for all $\mathrm{x} \in X$

(i) $\quad \mathrm{I}(\mathrm{x}, \alpha, \mu) \geq 0$.

(ii) $\quad I(x, \alpha \vee \gamma, \mu) \leq \mathrm{I}(\mathrm{x}, \alpha$, ? $)+\mathrm{I}(\mathrm{x}, \gamma$, ? $)$.

Proof: See, [1] and [3].

Theorem 2.5 If $\alpha$ is a finite measurable partition of $X$ with $I(x, \alpha$, ? $)<\infty$, for each $\mathrm{x} \in X$ then, $\lim _{n \rightarrow \infty} \frac{1}{n} I\left(x, \bigvee_{i=0}^{n-1} T^{-i} \alpha, \mu\right)$, for each $x \in X$ exists and is equal to infimum.

Proof: Write $a_{n}=I\left(x, \bigvee_{i=0}^{n-1} T^{-i} \alpha, \mu\right)$, for each $x \in X$. Clearly, the sequence $\left(a_{n}\right)_{n \geq 1}$ satisfies the conditions of Lemma 2.3and Theorem 2.4. Thus one has only the Lemma $2.3 \lim _{n \rightarrow \infty} \frac{1}{n} I\left(x, \bigvee_{i=0}^{n-1} T^{-i} \alpha, \mu\right)$, for each $\mathrm{x} \in X$ exists and is equal to the infimum.

Definition 2.6 Let $\alpha$ be a finite measurable partition of the dynamical system $(X, T)$ with $\mathrm{I}(\mathrm{x}, \alpha$, $\mu)<\infty$, for each $\mathrm{x} \in X$ then the limit function $I(x, T, \alpha, \mu)=\lim _{n \rightarrow \infty} \frac{1}{n} I\left(x, \bigvee_{i=0}^{n-1} T^{-i} \alpha, \mu\right)$, for each $\mathrm{x} \in X$ is called the local information function of $T$ with respect to finite measurable partition $\alpha$.

Definition 2.7 Let $\alpha$ be a finite measurable partition of $X$ with $\mathrm{I}(\mathrm{x}, \alpha, \mu)<\infty$, for all $\mathrm{x} \in X$, the quantity

$\mathrm{I}(\mathrm{x}, T, \mu)=\sup _{\alpha}\{\mathrm{I}(x, T, \alpha, \mu) \mid \alpha$ is a finite measurable partition of $X$ with $\mathrm{I}(\mathrm{x}, \alpha$, ? $)<\infty\}$

is called the local information function of dynamical system $(X, T)$. Where the supremum is taken over all finite measurable partitions of $X$ with the finite information functions.

Definition 2.8 Let $\alpha$ be a finite measurable partition of $X$ with $\mathrm{I}(\mathrm{x}, \alpha, \mu)<\infty$, for each $\mathrm{x} \in X$. Then, if $\bigvee_{i=-\infty}^{\infty} T^{i} \alpha=\beta$, then the partition $\alpha$ is called a generator of the $\sigma-$ algebra $\beta$ for T.

Theorem 2.9 Let $\alpha$ be a generating measurable partition of the dynamical system $(X, T)$ with $\mathrm{I}(\mathrm{x}, \alpha$, ? $)<\infty$, for each $x \in X$. Then, for all $x \in X$,

Proof: See, [1] and [3].

$$
I(x, T, \mu)=I(x, T, \alpha, \mu) \text {. }
$$

In the following Theorem we will prove the local information function of the dynamical system $(X, T)$ is affine.

Theorem 2.10 The local information function of the dynamical system $(X, T)$ is affine, i.e.

for each pair $\mu_{1}, \mu_{2} \in M(X, T)$ and $\lambda \in[0,1]$.

$$
I\left(x, T, \lambda \mu_{1}+(1-\lambda) \mu_{2}\right)=\lambda I\left(x, T, \mu_{1}\right)+(1-\lambda) I\left(x, T, \mu_{2}\right) .
$$

Proof: Let $\alpha$ be a finite measurable partition of $X$,then

$$
I\left(x, \alpha, \lambda \mu_{1}+(1-\lambda) \mu_{2}\right) \geq \lambda I\left(x, \alpha, \mu_{1}\right)+(1-\lambda) I\left(x, \alpha, \mu_{2}\right)
$$


The "concavity" inequality (2.1) is a direct consequence of the definition of $I(x, \alpha, \mu)$ and the "concavity" of the function $x \rightarrow-\log x$. Conversly, one has inequalities

and

$$
-\log \left(\lambda \mu_{1}\left(A_{i}\right)+(1-\lambda) \mu_{2}\left(A_{i}\right)\right) \leq-\log \lambda-\log \mu_{1}\left(A_{i}\right),
$$

because $x \rightarrow-\log x$ is decreasing. So we have,

$$
-\log \left(\lambda \mu_{1}\left(A_{i}\right)+(1-\lambda) \mu_{2}\left(A_{i}\right)\right) \leq-\log (1-\lambda)-\log \mu_{2}\left(A_{i}\right),
$$

$$
\begin{aligned}
& -\sum_{i=1}^{n} \chi_{A_{i}}(x) \log \left(\lambda \mu_{1}\left(A_{i}\right)+(1-\lambda) \mu_{2}\left(A_{i}\right)\right) \\
& \leq-\lambda \log \lambda-(1-\lambda) \log (1-\lambda)+\lambda I\left(x, \alpha, \mu_{1}\right)+(1-\lambda) I\left(x, \alpha, \mu_{2}\right) .
\end{aligned}
$$

Therefore one obtains the "convexity" bound

$$
\begin{aligned}
I\left(x, \alpha, \lambda \mu_{1}+\right. & \left.(1-\lambda) \mu_{2}\right) \\
& \leq-\lambda \log \lambda-(1-\lambda) \log (1-\lambda)+\lambda I\left(x, \alpha, \mu_{1}\right)+(1-\lambda) I\left(x, \alpha, \mu_{2}\right)
\end{aligned}
$$

now replacing $\alpha$ by $\bigvee_{i=0}^{n-1} T^{-i} \alpha$ in (2.1), dividing by $n$ and taking $\lim _{n \rightarrow \infty}$ gives

Similarly from (2.2), since

$$
I\left(x, T, \alpha, \lambda \mu_{1}+(1-\lambda) \mu_{2}\right) \geq \lambda I\left(x, T, \alpha, \mu_{1}\right)+(1-\lambda) I\left(x, T, \alpha, \mu_{2}\right) .
$$

$$
\frac{-(\lambda \log \lambda+(1-\lambda) \log (1-\lambda))}{n} \rightarrow 0 \text { as } n \rightarrow \infty,
$$

one deduce the converse inequality

$$
I\left(x,, T, \alpha, \lambda \mu_{1}+(1-\lambda) \mu_{2}\right) \leq \lambda I\left(x, T, \alpha, \mu_{1}\right)+(1-\lambda) I\left(x, T, \alpha, \mu_{2}\right) .
$$

Hence one concludes that the map $\mu \rightarrow I(x, T, \alpha, \mu)$ is affine, i.e.

$$
I\left(x, T, \alpha, \lambda \mu_{1}+(1-\lambda) \mu_{2}\right)=\lambda I\left(x, T, \alpha, \mu_{1}\right)+(1-\lambda) I\left(x, T, \alpha, \mu_{2}\right)
$$

For each partition $\alpha$, each pair $\mu_{1}, \mu_{2} \in M(X, T)$ and $\lambda \in[0,1]$. Finally it follows from the Theorem 2.9 that the local information function of the dynamical system $(X, T)$ is affine,

$I\left(x, T, \lambda \mu_{1}+(1-\lambda) \mu_{2}\right)=\lambda I\left(x, T, \mu_{1}\right)+(1-\lambda) I\left(x, T, \mu_{2}\right)$.

We recall some classical results that we need in the sequel.

Theorem 2.11 (Choquet) Suppose that $\mathrm{Y}$ is a compact convex metrisable subset of a locally convex space $E$, and that $x_{0} \in Y$. Then, there exists a probability measure $\tau$ on $Y$ which represents $x_{0}$ and is supported by the extreme points of $\mathrm{Y}$, that is, $\varphi\left(x_{0}\right)=\int_{Y} \varphi d \tau$ for every continuous linear functional $\varphi$ on $\mathrm{E}$, and $\tau(\operatorname{ext}(Y))=1$.

Proof: See Phelps [6] for a proof of Choquet's theorem.

Let $\mu \in M(X, T)$ and $f: X \rightarrow \mathbb{R}$ be a bounded measurable function. As we know that $E(X, T)$ equals the extreme points of $M(X, T)$, applying Choquet's Theorem for $\mathrm{E}=\Omega(X)$, the space of finite regular Borel measures on $X$, and $Y=M(X, T)$, andusing the linear functional $\varphi: \Omega(X) \rightarrow \mathbb{R}$ given by

$\varphi(\mu)=\int_{X} f d \mu$, we have the following corollary.

Corollary2.12 Suppose that $T: X \rightarrow X$ is a continuous map on the compact metric space $X$. Then, for each $\mu \in M(X, T)$, there is a unique measure $\tau$ on the Borel subsets of the compact metrisable space $M(X, T)$, such that $\tau(E(X, T))=1$ and

$$
\int_{X} f(x) d \mu(x)=\int_{E(X, T)}\left(\int_{X} f(x) d m(x)\right) d \tau(m)
$$

for every bounded measurable function $f: X \rightarrow \mathbb{R}$.

Under the assumption of Corollary 2.12, we write $\mu=\int_{E(X, T)} m d \tau(m)$, called the ergodic decomposition of $\mu$.

\section{WEIGHTED INFORMATION FUNCTION OFDYNAMICAL SYSTEMS}

In this section, we introduce the concept of weighted information function for a continuous map $T: X \rightarrow X$ on a compact metric space $\mathrm{X}$ with finite local information function, and state some of its properties. 
Definition 3.1Suppose that $T: X \rightarrow X$ is a continuous map on the compact topological space $X$, and $\mu \in M(X, T)$ be such that $\mathrm{I}(\mathrm{x},, T, \mu)<\infty$, for $\in X$. The weighted information function of $T$ (with respect to $\mu), L_{T}(., \mu): C(X) \rightarrow \mathbb{R}$, is defined as

$$
L_{T}(f, \mu):=\int_{X} f(x) \quad I(x, T, \mu) d \mu(x)
$$

for all $f \in C(X)$. In other words, the weighted information function is an integral function. Theorem 3.2Suppose that $T: X \rightarrow X$ is a continuous map on the compact metric space $\mathrm{X}$ such that $I(., T, \mu)<\infty$. Then,

(i) Given any $\mu \in M(X, T)$, the weighted information function $f \rightarrow L_{T}(f, \mu)$ is linear.

(ii) If $\mu \in M(X, T)$ and $\mu=\int_{E(X, T)} m d \tau(m)$ is the ergodic decomposition of $\mu$, then,

for all $f \in C(X)$.

$$
L_{T}(f, \mu)=\int_{E(X, T)} L_{T}(f, m) d \tau(m)
$$

Proof : Note that the condition $I(., T, \mu)<\infty$ guarantees that $L_{T}(f, \mu)$ is well-defined for all $\mu \in$ $M(X, T)$.

(i)Obvious.

(ii) We have

For $f \in C(X)$.

$$
\begin{aligned}
L_{T}(f, \mu) & =\int_{X} f(x) I(x, T, \mu) d \mu(x) \\
& =\int_{E(X, T)}\left(\int_{X} f(x) I(x, T, m) d m(x)\right) d \tau(m) \\
& =\int_{E(X, T)} L_{T}(f, m) d \tau(m) .
\end{aligned}
$$

Theorem 3.3 Let $\alpha$ be a measurable partition of the dynamical system $(X, T)$ with $I(x, T, \alpha, \mu)<\infty$, for

$x \in X$. Then for every $k \in N$,

Proof: We obtain immediately

$$
I(x, T, \alpha, \mu)=I\left(x, T, \bigvee_{j=0}^{k} T^{-j}(\alpha), \mu\right)
$$

$$
\begin{gathered}
I\left(x, T \bigvee_{j=0}^{k} T^{-i}(\alpha), \mu\right)=\lim _{n \rightarrow \infty} \frac{1}{n} I\left(x, \bigvee_{i=0}^{n-1} T^{-i}\left(\bigvee_{j=0}^{k} T^{-j}(\alpha)\right), \mu\right) \\
=\lim _{n \rightarrow \infty} \frac{1}{n} I\left(x, \bigvee_{t=0}^{n+k-1} T^{-t}(\alpha), \mu\right) \\
=\lim _{p \rightarrow \infty} \frac{p}{p-k} \frac{1}{p} I\left(x, \bigvee_{t=0}^{p-1} T^{-t}(\alpha), \mu\right) \\
=I(x, T, \alpha, \mu) .
\end{gathered}
$$

Theorem 3.4 Let $\alpha$ be a measurable partition of the dynamical system $(X, T)$ with $I(x, T, \alpha, \mu)<\infty$, for

$x \in X$. Then for every $k \in N$,

Proof:By Theorem 3.3, we have

$$
I\left(x, T^{k}, \mu\right)=k I(x, T, \mu)
$$

$$
I\left(x, T^{k}, \alpha, \mu\right)=I\left(x, T^{k}, \bigvee_{i=0}^{n-1} T^{-i}(\alpha), \mu\right)
$$




$$
\begin{gathered}
=\lim _{n \rightarrow \infty} \frac{1}{n} I\left(x, \bigvee_{j=0}^{n-1} T^{-j k}\left(\bigvee_{i=0}^{k-1} T^{-i}(\alpha)\right), \mu\right) \\
=\lim _{n \rightarrow \infty} \frac{1}{n} I\left(x, \bigvee_{i=0}^{n k-1} T^{-i}(\alpha), \mu\right) \\
=\operatorname{kim}_{n \rightarrow \infty} \frac{1}{n k} I\left(x, \bigvee_{i=0}^{n k-1} T^{-i}(\alpha), \mu\right) \\
=k I(x, T, \alpha, \mu) .
\end{gathered}
$$

Theorem 3.5Suppose that $T: X \rightarrow X$ is a continuous map on the compact metric space $X$,and $\mu \in$ $M(X, T)$. Then for every $k \in N$,

$$
L_{T^{k}}(f, \mu)=k L_{T}(f, \mu) .
$$

Proof: By Theorem 3.4 we know that for each $x \in X$ and $k \in N$,

$$
I\left(x, T^{k}, \mu\right)=k I(x, T, \mu)
$$

So, if $f \in C(X)$, then,

Therefore,

$$
f(x) I\left(x, \alpha, T^{k}, \mu\right)=f(x) k I(x, T, \alpha, \mu)
$$

$$
\begin{array}{r}
L_{T^{k}}(f, \mu)=\int_{X} f(x) I\left(x, T^{k}, \mu\right) d \mu(x) \\
=\int_{X} k f(x) I(x, T, \mu) d \mu(x) \\
=k L_{T}(f, \mu)
\end{array}
$$

Theorem 3.6Suppose that $I d: X \rightarrow X$ is the identity map on the compact metric space $X$, and $\mu \in M(X, T)$. Then, $L_{I d}(f, \mu)=0$.

Proof: Since $I(x, I d, \mu)=0$, so $L_{I d}(f, \mu)=0$.

Theorem 3.7 If $T_{1}: X_{1} \rightarrow X_{1}$ and $T_{2}: X_{2} \rightarrow X_{2}$ are topologically isomorphic continuous maps via the homeomorphism $\varphi: X_{1} \rightarrow X_{2}$, and $\mu \in M\left(X_{1}, T_{1}\right)$, then,

for all $f \in C\left(X_{1}\right)$.

$$
L_{T_{1}}(f, \mu)=L_{T_{2}}\left(f \varphi^{-1}, \mu \varphi^{-1}\right)
$$

proof: First, we prove that,

$$
I\left(x, T_{1}, \mu\right)=I\left(\varphi(x), T_{2}, \mu\right) .
$$

Let $\left(X_{1}, T_{1}\right)$ and $\left(X_{2}, T_{2}\right)$ be two isomorphic dynamical systems with $\varphi: X_{1} \rightarrow X_{2}, \varphi\left(T_{1}(x)\right)=$ $T_{2}(\varphi(x))$ for all $x \in X$. If $\alpha$ is a finite measurable partition of the dynamical system $\left(X_{1}, T_{1}\right)$ with $I\left(x, T_{1}, \alpha, \mu\right)<\infty$, for $x \in X$. Then, $\varphi(\alpha)$ is a finite measurable partition of the dynamical system $\left(X_{2}, T_{2}\right)$ with $I(y, T, \varphi(\alpha), \mu)<\infty$, for $\varphi(x)=y \in X_{2}$. Therefore for all $x \in X_{1}$,

$$
\begin{aligned}
I\left(y, T_{2}, \varphi(\alpha), \mu\right) & =I\left(y, \varphi o T_{1} o \varphi^{-1}, \varphi(\alpha), \mu\right) \\
& =\lim _{n \rightarrow \infty} \frac{1}{n} I\left(y, \bigvee_{i=0}^{n-1}\left(\varphi o T_{1}^{-i} o \varphi^{-1}\right) \varphi(\alpha), \mu\right) \\
=\lim _{n \rightarrow \infty} \frac{1}{n} I\left(\varphi^{-1}(y), \varphi^{-1}\left(\mathrm{~V}_{i=0}^{n-1} \varphi o T_{1}^{-i}(\alpha)\right)\right. & \\
& =\lim _{n \rightarrow \infty} \frac{1}{n} I\left(x, \bigvee_{i=0}^{n-1} T_{1}^{-i}(\alpha), \mu\right) \\
& =I\left(x, T_{1}, \alpha, \mu\right) .
\end{aligned}
$$

Let $\mu \in M\left(X_{1}, T_{1}\right)$, and $f \in C(X)$. Then,

$$
\begin{aligned}
L_{T_{l}}(f, \mu)=\int_{X_{1}} f(x) I\left(x, T_{1}, \mu\right) d \mu(x) \\
\quad=\int_{X_{I}} f(x) I\left(\varphi(x), T_{2}, \mu\right) d \mu(x)
\end{aligned}
$$




$$
\begin{aligned}
& =\int_{X_{2}} f\left(\varphi^{-1}(x)\right) I\left(x, T_{2}, \mu\right) d \mu\left(\varphi^{-1}(x)\right) \\
& =L_{T_{2}}\left(f \varphi^{-1}, \mu \varphi^{-1}\right) .
\end{aligned}
$$

\section{CONCLUSION}

For the classification of the dynamical systems based on isomorphism, isomorphism invariants play an important role. In this paper, we have introduced the weighted information function for dynamical systems compact metric spaces. It is a linear functional on $C(X)$. It is indeed an integral function. The map $I(x, T, \mu)$ is indeed a local information map and so the value $L_{T}(f, \mu)$ may be interpreted as the average information of $T$ weightened by $f$. We also generalized the invariance of the local information function of a system, under topological isomorphism, to the weighted information function.

\section{REFERENCES}

1. J. R.Brown, Ergodic theory and Topological Dynamics, Academic Pres, New York, (1976).

2. M. Ebrahimi, U. Mohamadi, $m$-Generators of fuzzy dynamical systems, Cankaya University Journal of Science and Engineering, 9 (2012), 167-182.

3. I. Guney, I. Tok and M. Yamankaradeniz, On the fuzzy local information function, Balkan Journal of Mathematics, 01 (2013) 44-60.

4. I. Tok, On the fuzzy local entropy function, E. U. J.Sci. Fac.Series A(Math), 44, (2005).

5. B. McMillan, The basic theorems of information theory, Anal. Math. Stat., 24, (1953), 196-219.

6. R. Phelps, Lectures on Choquets Theorem, D. Van Nostrand Co., Inc., Princeton, N. J.-Toronto, Ont.-London, (1966).

7. C. Shannon, A mathematical theory of communication. Bell Syst Tech Journal, 27 (1948), 379-423.

8. P. Walters, An Introduction to Ergodic Theory, Springer Verlag, (1982).

9. A. Zamani Bahabadi, Another proof for existence of dominated splitting for robustly ergodic

diffieomorphisms, Journal of mathematics and computer science, 6(2013), 13-17. 